\title{
Comparative Analysis of Surgical Outcomes of C1-2 Fusion Spine Surgery between Intraoperative Computed Tomography Image Based Navigation-Guided Operation and Fluoroscopy-Guided Operation
}

\author{
Jun Seok Lee, ${ }^{1-3}$ Dong Wuk Son, ${ }^{1-3}$ Su Hun Lee, ${ }^{1-3}$ Sung Soon $\mathrm{Ki}_{,}^{1-3}$ Sang Weon Lee, ${ }^{1-3}$ Geun Sung Song ${ }^{1-3}$ \\ Department of Neurosurgery, Pusan National University Yangsan Hospital, Yangsan, Korea \\ Research Institute for Convergence of Biomedical Science and Technology, ${ }^{2}$ Pusan National University Yangsan Hospital, Yangsan, Korea \\ Department of Neurosurgery, ${ }^{3}$ School of Medicine, Pusan National University Yangsan Hospital, Yangsan, Korea
}

Objective : Fixation of the $\mathrm{C} 1-2$ segment is challenging because of the complex anatomy in the region and the need for a high degree of accuracy to avoid complications. Preoperative 3D-computed tomography (CT) scans can help reduce the risk of complications in the vertebral artery, spinal cord, and nerve roots. However, the patient may be susceptible to injury if the patient's anatomy does not match the preoperative CT scans. The intraoperative 3D image-based navigation systems have reduced complications in instrument-assisted techniques due to greater accuracy. This study aimed to compare the radiologic outcomes of C1-2 fusion surgery between intraoperative CT image-guided operation and fluoroscopy-guided operation.

Methods : We retrospectively reviewed the radiologic images of 34 patients who underwent C1-2 fusion spine surgery from January 2009 to November 2018 at our hospital. We assessed 17 cases each of degenerative cervical disease and trauma in a study population of 18 males and 16 females. The mean age was 54.8 years. A total of 139 screws were used and the surgical procedures included 68 screws in the C1 lateral mass, 58 screws in C2 pedicle, nine screws in C2 lamina and C2 pars screws, four lateral mass screws in sub-axial level. Of the 34 patients, 19 patients underwent screw insertion using intraoperative mobile CT. Other patients underwent atlantoaxial fusion with a standard fluoroscopy-guided device.

Results : A total of 139 screws were correctly positioned. We analyzed the positions of 135 screws except for the four screws that performed the lateral mass screws in C3 vertebra. Minor screw penetration was observed in seven cases (5.2\%), and major pedicle screw penetration was observed in three cases (2.2\%). In one case, the malposition of a C2 pedicle screw was confirmed, which was subsequently corrected. There were no complications regarding vertebral artery injury or onset of new neurologic deficits. The screw malposition rate was lower (5.3\%) in patients who underwent intraoperative CT-based navigation than that for fluoroscopy-guided cases (10.2\%). And we confirmed that the operation time can be significantly reduced by surgery using intraoperative $\mathrm{O}$-arm device.

Conclusion : Spinal navigation using intraoperative cone-beam CT scans is reliable for posterior fixation in unstable C1-2 pathologies and can be reduced the operative time.

Key Words : Cervical vertebrae · Arthrodesis · Dimensional measurement accuracy · Atlanto-axial joint.

- Received : August 10, 2019 •Revised : September 26, 2019 •Accepted : November 15, 2019

- Address for reprints : Dong Wuk Son

Department of Neurosurgery, Pusan National University Yangsan Hospital, 20 Geumo-ro, Mulgeum-eup, Yangsan 50612, Korea

Tel : +82-55-360-2126, Fax : +82-55-360-2156, E-mail : md6576@naver.com, ORCID : https://orcid.org/0000-0002-9154-1923

This is an Open Access article distributed under the terms of the Creative Commons Attribution Non-Commercial License (http://creativecommons.org/licenses/by-nc/4.0) which permits unrestricted non-commercial use, distribution, and reproduction in any medium, provided the original work is properly cited. 


\section{INTRODUCTION}

The atlantoaxial junction is a specialized region that plays an important role in the axial rotation of the cervical vertebrae. It is vulnerable to inflammatory disorders, trauma, and neoplasms. These diseases can destroy adjacent neurovascular structures and lead to upper cervical spinal instability and neurologic deficits.

Arthrodesis of the atlantoaxial junction is a challenging procedure because the upper cervical spine has a highly variable pedicle anatomy and vertebral artery anomalies. These anatomically variable neurovascular structures make pedicle screw fixations technically difficult ${ }^{16)}$. Previous studies have reported that the incidence of vertebral artery injuries is $0-8.2 \%$ in atlantoaxial fusion surgery ${ }^{12}$. To avoid the catastrophic complications related to surgery, various techniques have been introduced to achieve upper cervical fixation ${ }^{4,5)}$. The posterior $\mathrm{C} 1$ lateral mass and $\mathrm{C} 2$ pedicle screw with polyaxial screws and rod fixation system is widely used and recognized as a conventional surgical method.

Recently, the development of the image guided navigation technology has led to significant changes in high-risk upper cervical surgery. The $\mathrm{O}$-arm imaging system (Medtronic, Inc, Mineapolis, MN, USA) is considered to be accurate for spinal surgery. According to literature, there are several benefits of using an intraoperative computed tomography (CT) guided navigation system for spinal surgery : multi planar images of different operative levels in a single sequence can be obtained which improves surgical accuracy, exposure to radiation is reduced, and screw positions can be checked in the surgical field which reduces the possibility of failure.

Several studies have described the usefulness of screw fixation using the intraoperative CT based navigation in the surgical field of atlantoaxial junction ${ }^{2,7,8,13)}$. These studies focused on the accuracy of the placement of pedicle screws and surgical complications and most have shown satisfactory results. However, few studies have evaluated the usefulness of conventional methods by using $\mathrm{C}$-arm fluoroscopy devices due to lack of samples for comparison. Our medical center had installed an intraoperative CT based navigation system in 2014 for spinal surgery. In this study, we analyzed the radiologic and operative parameters associated with surgery of an unstable atlantoaxial junction. Additionally, we compared the differences between surgical results and confirmed the useful- ness of atlantoaxial fusion surgery by using intraoperative CT based navigation devices.

\section{MATERIALS AND METHODS}

\section{Patient demographics}

The study protocol was approved by the Institution Review Board of Pusan National University Yangsan Hospital, which waived the requirement for informed consent due to the retrospective nature of this study (IRB No. 05-2019-117). We retrospectively reviewed the medical records of 34 patients who underwent atlantoaxial fusion between January 2009 and November 2018. All the patients were treated for correction of atlantoaxial instability by one experienced surgeon and two beginner surgeons. The mean age was 54.81 years (range, 17-80). All the patients had different causes for atlantoaxial instability. Rheumatoid arthritis, odontoid fracture, and transverse ligament injury were the most common causes for cervical instability. Of the 34 patients, 17 were caused by trauma and the rest were degenerative cases. The radiologic examinations included CT of cervical spine, plain radiography, flexion/extension radiography for cervical instability and cervical magnetic resonance tomography. For identification of the patency of vertebral artery, a 3D reconstruction CT was performed preoperatively. All the radiologic examinations were performed preoperatively.

The patients were divided into two groups based on the imaging technique used for screw insertion. The C-arm group used conventional fluoroscopic imaging during screw insertion and the $\mathrm{O}$-arm group used intraoperative navigation devices during surgery. Our institution used an $\mathrm{O}$-arm intraoperative cone-beam CT scanner connected to an S7 Stealthstation surgical navigation system (Medtronic, Inc).

The clinical factors included the patient's age, gender, body mass index, and medical history. The intraoperative parameters included the operation time, intraoperative estimated blood loss (EBL), transfusion of blood products, and operation related complications. The operative data were collected from the anesthesia records. The patient demographics and operative data are summarized in Table 1. 
Table 1. Comparison of the patients' demographic characteristics

\begin{tabular}{|c|c|c|c|}
\hline & C-arm group $(n=15)$ & 0 -arm group $(n=19)$ & $p$-value \\
\hline \multicolumn{4}{|l|}{ Clinical parameters } \\
\hline Age & $49.93 \pm 18.08$ & $58.37 \pm 15.38$ & 0.161 \\
\hline Gender (male : female) & $11: 4$ & $7: 12$ & 0.171 \\
\hline BMI & $22.05 \pm 2.26$ & $24.74 \pm 3.64$ & 0.013 \\
\hline HT & $1 / 15(6.7)$ & $9 / 19(47.0)$ & 0.020 \\
\hline DM & $1 / 15(6.7)$ & 4/19 (21.1) & 0.355 \\
\hline Rheumatoid arthritis & $1 / 15(6.7)$ & $4 / 19(21.1)$ & 0.355 \\
\hline Trauma : degeneration & $10: 5$ & $8: 11$ & 0.185 \\
\hline \multicolumn{4}{|l|}{ Operative parameters } \\
\hline EBL & $796.67 \pm 461.55$ & $621.82 \pm 553.94$ & 0.312 \\
\hline Operative time & $372.00 \pm 73.53$ & $319.74 \pm 62.35$ & 0.036 \\
\hline Transfusion rate & $4 / 15(26.7)$ & $8 / 19(42.1)$ & 0.476 \\
\hline Fusion rate & 15/15 (100.0) & $18 / 19(94.7)$ & $>0.999$ \\
\hline \multicolumn{4}{|l|}{ Anatomical variation } \\
\hline HRVA & $7 / 15(46.7)$ & $6 / 19(31.6)$ & 0.484 \\
\hline Median shifting & $3 / 15(20.0)$ & $2 / 19(10.5)$ & 0.634 \\
\hline \multicolumn{4}{|l|}{ Average of pedicle size (mm) } \\
\hline Right & 4.17 & 4.93 & \\
\hline Left & 4.93 & 4.56 & \\
\hline Mean pedicle size (mm) & $4.55 \pm 1.01$ & $4.70 \pm 1.45$ & 0.637 \\
\hline $\begin{array}{l}\text { Numbers of narrow pedicle size }(<4 \text { mm pedicle } \\
\text { size) }\end{array}$ & $8 / 30(26.7)$ & $7 / 34(20.6)$ & 0.768 \\
\hline Total numbers of patients with anomaly & $9 / 15(60.0)$ & 9/19 (47.3) & 0.510 \\
\hline
\end{tabular}

Values are presented as mean \pm standard deviation or number (\%) unless otherwise indicated. BMI : body mass index, HT : hypertension, DM : diabetes mellitus, EBL : estimated blood loss, HRVA : high riding vertebral artery

\section{Surgical technique and planning of C2 screw fixation}

All the procedures were performed using the conventional Goel and Harms technique of $\mathrm{C} 1$ lateral mass screw and C2 pedicle screw fixation. The patients were placed in a prone position with a radiolucent Mayfield fixator to secure the head and a dissection of the posterior region of the C1-2 complex was performed to check the anatomical landmarks and mount the navigational reference frame on the $\mathrm{C} 2$ spinous process. Next, an O-arm scan was performed intraoperatively and the scanned images were directly transferred with the navigation devices and auto-regulation was performed. This process took about 10 minutes to acquire the image and register, which took lesser time and effort when compared to the surface matching method of registration. A small radiopaque screw was inserted at the entry point of the $\mathrm{C} 2$ pedicle screw to confirm the accuracy of the images and a navigation probe was placed on the small screw to check whether correct images appeared on the navigation screen. The $\mathrm{C} 2$ nerve root in the $\mathrm{C} 1$ lateral mass screw fixation was saved to reduce the postoperative occipital neuralgia in most cases. If the patient had a poor bony quality, additional fusion space was obtained on C1-2 articular space by cutting the $\mathrm{C} 2$ root. $\mathrm{C} 2$ pedicle screw fixations were performed if the width of the $\mathrm{C} 2$ pedicle was greater than $4 \mathrm{~mm}$ in the preoperative axial image, even if there was a high riding vertebral artery. If the size of the pedicle was under $4 \mathrm{~mm}$, the insertion of the pedicle screw was determined based on the presence of the anomaly of the $\mathrm{C} 2$ vertebra. An alternative $\mathrm{C} 2$ screw method such as a pars screw or a lamina screw was considered if the pedicle size was less than 3 
$\mathrm{mm}$ in size. If the mean cortex depth of C2 lamina was more than $3 \mathrm{~mm}$, lamina screw was selected. If the cortex depth of C2 lamina is not thick enough, the pars screw was chosen as second choice. A pars screw uses a shorter screw than a pedicle screw, but has sufficient pull out strength and the screw location could be confirmed in in advance with navigation devices. Fig. 1 shows the flow chart for the choice of the C2 screw method. After the screw fixation, the intraoperative $\mathrm{O}$-arm CT was re-examined at the operative site to confirm if the screw was well positioned. And the posterior arch of $\mathrm{Cl}$ and C2 lamina were decorticated and mixture of demineralized bone matrix and autogenous bone graft taken from the posterior iliac crest were placed on the bony fusion bed.

\section{Assessment of screw positioning}

Postoperative CT images were taken 1 year after the atlantoaxial fusion to confirm the position of the screws and verify spinal bony fusion. The radiology images were analyzed by two independent spine surgeons who were not involved in the study and blinded to all clinical information. The screw placement was classified according to the cervical classification system described previously. The grading system described by Gertzbein and Robbins ${ }^{3)}$ was used. Grade 0, when a screw was placed inside the bone; grade I, screw perforation of the cortex within $2 \mathrm{~mm}$; grade II, screw perforation from $2 \mathrm{~mm}$ to 4 $\mathrm{mm}$; and grade III, screw perforation of more than $4 \mathrm{~mm}$.
Grade I was recognized as minor cortical penetration and grade II and III were recognized as major cortical penetration because of the potential of neurovascular injury.

\section{Statistical analysis}

The statistical analyses were performed using SPSS version 18.0 (SPSS, INC., Chicago, IL, USA). A $p$ value $<0.05$ was considered significant. The normally distributed data were compared using the unpaired t test. The Fisher's exact test and chisquare test were used to analyze the categorical data.

\section{RESULTS}

The preoperative CT examination showed the anatomical abnormalities associated with the atlantoaxial fusion surgery. A narrow pedicle, high riding vertebral artery, and medial shift of the vertebral artery were defined as anomalies associated with the surgical procedures ${ }^{10)}$. If the prominent part of intra-axial vertebral artery was located within the vertical line of C3 transverse foramen on coronal CT scan, it was defined as medial shifting. If the $\mathrm{C} 2$ pedicle diameter was less than 4 $\mathrm{mm}$, it was defined as a narrow pedicle. The average width of the $\mathrm{C} 2$ pedicle was $4.55 \mathrm{~mm}$ for the C-arm group and 4.75 $\mathrm{mm}$ for the $\mathrm{O}$-arm group. Among the patients in the $\mathrm{C}$-arm group, a high riding vertebral artery was observed in seven

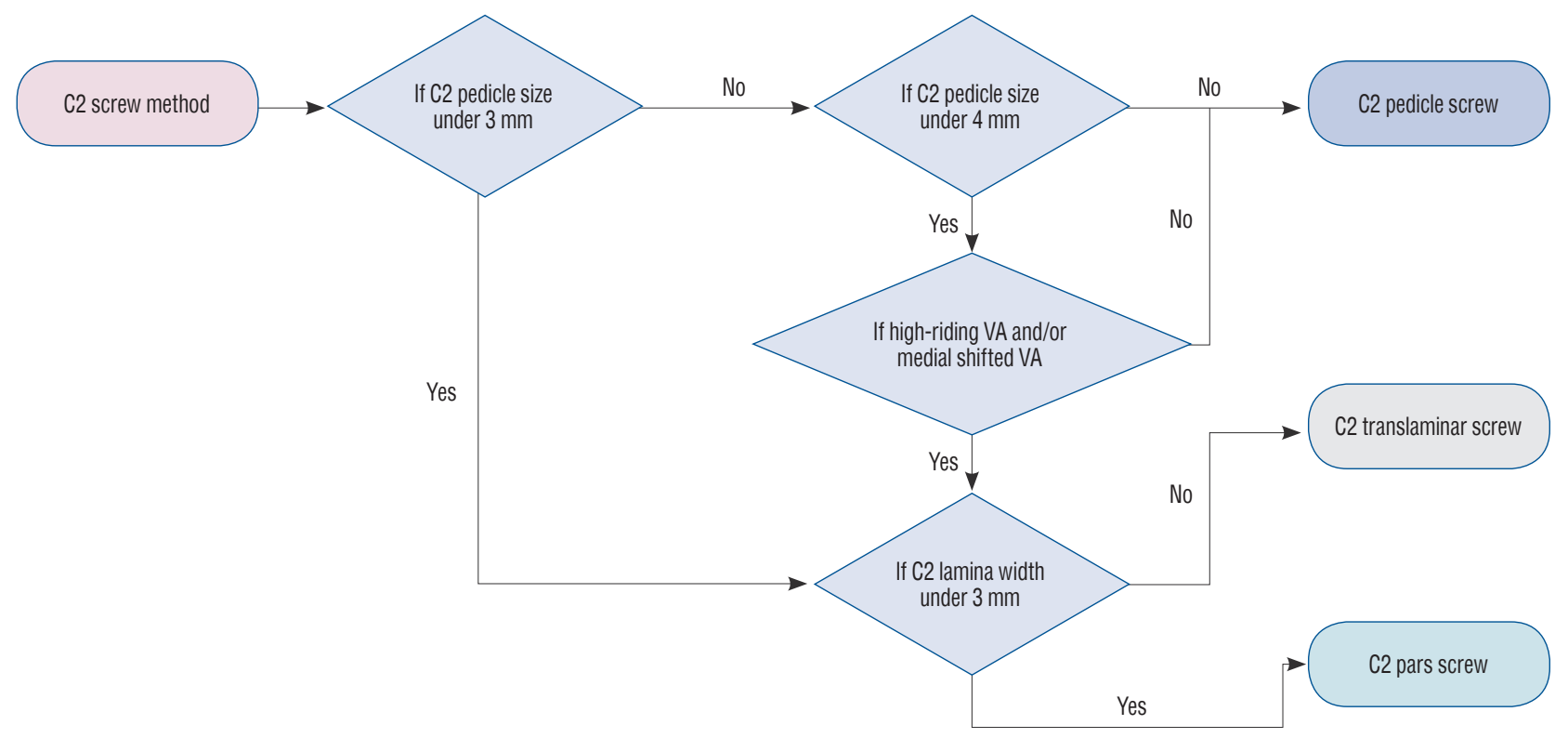

Fig. 1. Schematic flow chart for the choice of the C2 vertebral screws. VA : vertebral artery. 
and a medial shift was observed in three patients. Additionally, the O-arm group consisted of six patients of vertebral artery and two patients with medial shift of the vertebral artery. Table 1 shows the abnormal C2 vertebral structures.

\section{Accuracy evaluation of pedicle screw placement}

A total of 139 screws were placed in 34 patients; 68 screws were placed in $\mathrm{C} 1$ vertebra, 67 in $\mathrm{C} 2$ vertebra, and four screws in the remaining levels. At the $\mathrm{C} 2$ vertebral level, according to the screw insertion methods, there were 58 cases with $\mathrm{C} 2$ pedicle screws, two with short pars screws and seven with lamina screws. In the O-arm group, if it was difficult to insert a C2 pedicle screw, placement of a C2 short pars screw was first tried followed by a lamina screw as a second choice. There was no nerve root injury or vascular complication due to the screw fixations. The types and locations of screws used during sur- gery are summarized in Table 2 .

We analyzed the positions of 135 screws except for the four screws that performed the lateral mass screws in C3 vertebra. Of the total 135 screws, 125 were classified as grade 0 , seven as grade I, 1 as grade II, and two as grade III. The authors regarded grade 0 and I as acceptable results. Conversely, screws of grade II and III may cause clinical problems and were considered as major cortical penetration. The screw accuracy rate of the O-arm group was $94.7 \%$ which was significantly than that for the C-arm group. Particularly, O-arm group showed an improvement in accuracy rate in the $\mathrm{C} 2$ vertebrae. This trend was also appeared with insertion of $\mathrm{C} 2$ pedicle screws, which actively used navigation instruments. The $\mathrm{O}$-arm group showed superior results even when it was limited to $\mathrm{C} 2$ pedicle screws. In patients with a C2 pedicle screw insertion with VA anomaly, there was no grade II or III malposition in $\mathrm{O}$-arm

Table 2. Types of the screw used during the surgery and their locations

\begin{tabular}{|c|c|c|c|}
\hline & C-arm group $(n=15)$ & O-arm group $(n=19)$ & Total $(n=34)$ \\
\hline C1 level & Lateral mass screws : 30 & Lateral mass screws : 38 & 68 \\
\hline \multirow[t]{3}{*}{ C2 level } & Pedicle screws : 26 & Pedicel screws : 32 & 58 \\
\hline & Short pars screws : 1 & Short pars screws : 1 & 2 \\
\hline & Laminar screws : 2 & Laminar screws : 5 & 7 \\
\hline Other levels & Lateral mass screws : 4 & None & 4 \\
\hline Total number of screws & 63 & 72 & 139 \\
\hline
\end{tabular}

Table 3. Distribution of screw position according to the grading system

\begin{tabular}{|c|c|c|c|c|}
\hline & Grade 0 & Grade I & Grade II & Grade III \\
\hline \multicolumn{5}{|c|}{ Accuracy of the positioning of all the screws in each group in $\mathrm{C} 1-2$ lesion } \\
\hline \multicolumn{5}{|l|}{ C-arm group (59 screws) } \\
\hline C1 (30 screws) & 28 & 1 & 0 & 1 \\
\hline C2 (29 screws) & 25 & 3 & 0 & 1 \\
\hline Accuracy rate (\%) & 89.8 & 6.8 & 0 & 3.4 \\
\hline \multicolumn{5}{|l|}{ O-arm group (76 screws) } \\
\hline C1 (38 screws) & 36 & 2 & 0 & 0 \\
\hline C2 (38 screws) & 36 & 1 & 1 & 0 \\
\hline Accuracy rate (\%) & 94.7 & 3.9 & 1.3 & 0 \\
\hline \multicolumn{5}{|c|}{ Accuracy of the positioning of the $C 2$ pedicle screws in each group } \\
\hline C-arm group (26 screws) & 23 & 2 & 0 & 1 \\
\hline Accuracy rate (\%) & 88.5 & 7.7 & 0 & 3.8 \\
\hline O-arm group (32 screws) & 30 & 1 & 1 & 0 \\
\hline Accuracy rate (\%) & 93.8 & 3.1 & 3.1 & 0 \\
\hline
\end{tabular}


group. However, rate of screw malposition was very low in both groups. Thus, we could not prove that the O-arm group was statistically superior to the screw accuracy over the C-arm group. The distribution of the accuracy of screw positions ac-
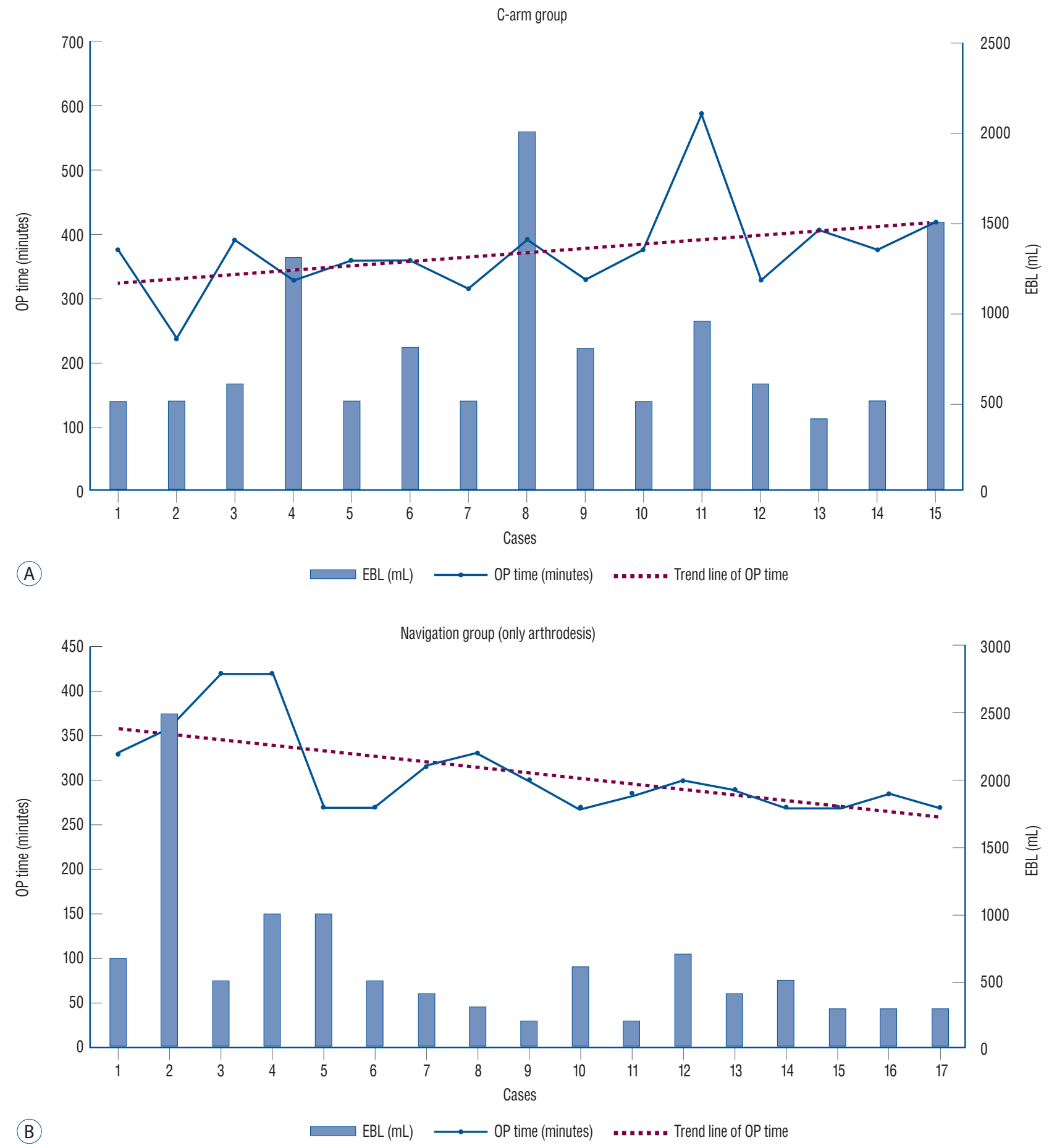

Fig. 2. Change in surgery results according to the accumulation of experience. $A$ : In the C-arm group, the estimated blood loss varied widely from case to case and the trend line of the operative time did not change much with time. B:In the O-arm group, the trend line of the operative time shows a decrease and estimated blood loss is stabilized to a certain level with the increase in the number of surgical cases. EBL : estimated blood loss, OP : operative time. 
cording to the grading system is summarized in Table 3.

\section{Analysis of operative factors}

There was a concern that surgery with the navigational device may take longer than with the fluoroscopic device. However, the authors took two images when working with the intraoperative CT based navigation device. One image was taken to register the images, and a second was taken to confirm the screw position after inserting the pedicle screw. Each image took about 10 additional minutes to record. However, according to the data, we can confirm that the operation time reduced significantly in the $\mathrm{O}$-arm group of patients. We identified that operation time could be significantly reduced when compared to the time taken to obtain the images when surgeons grasped the anatomical structure through the 3-D images. One notable feature of the operation time analysis is the fact that the operation time for simple cases that did not need neural decompression decreased over time. As the experience of the surgeons increased, it is assumed that this result was achieved due to familiarity with the O-arm machine. A flow chart on the change of operative factors according to increased experiences is illustrated in Fig. 2.

Bone fusion was defined when the atlantodental interval did not change and the inter-spinous distance decreased below $2 \mathrm{~mm}$ in the postoperative dynamic X-ray. When the halo sign was observed in postoperative CT images, it was defined as non-fusion, which was present in only one patient in the Oarm group. Bone fusion was successfully accomplished in most patients.

The average EBL in the $\mathrm{O}$-arm group was lower than that in the C-arm group. However, there were significant differences in EBL between the cases and not statistically results. The EBL was significantly higher in patients who required additional decompression due to spinal stenosis.

\section{Analysis of surgical complications}

No serious complications such as vascular complication, nerve root injury, or spinal cord injury occurred in both groups. However, one case from the $\mathrm{O}$-arm group was found to have abnormal $\mathrm{C} 2$ pedicle screw position during a scan of the cervical spine which was removed and converted to a lamina screw. The patient did not have any specific neurological abnormality postoperatively. This complicated case is illustrated in Fig. 3. In the C-arm group, two patients complained of postoperative continuous occipital neuralgia and one patient had additional antibiotic treatment after wound infection. Additionally, in $\mathrm{O}$-arm group, three patients underwent additional local block due to postoperative occipital neuralgia and one patient was diagnosed with meningitis. There were no serious postoperative complications in both groups.

\section{Illustrated image of the complicated case}

A 66-year-old woman with neck pain and headache for the past 7 months visited our clinic. On magnetic resonance imaging (MRI) examination, pannus and soft tissue swellings were observed around the odontoid process and right facet joint. On CT, atlantoaxial instability and severe sclerotic changes were identified in the C1-2 complex (Fig. 3A). The preoperative MRI showed a diffuse soft tissue lesion (pannus) around the odontoid process with enhancement. Surgery was performed with the Harm's technique. After screw fixation, an intraoperative CT confirmed that the right $\mathrm{C} 2$ pedicle invaded into the spinal canal. The right C2 pedicle screw was removed and an alternative C2 lamina screw was inserted (Fig. 3C). After the procedure, the patient recovered fully without any signs of neurologic deficit. No abnormal findings were observed on the X-ray after 3 months.

\section{DISCUSSION}

High cervical spine fusion surgery is difficult; surgeons must identify the anomaly in the C2 vertebrae and make a plan prior to surgery. The most common anatomical variations are high-riding vertebral arteries and narrow pedicle sizes. In the present study, the ratio of patients with abnormal high-riding vertebral artery and narrow pedicle size $(<4 \mathrm{~mm})$ was more than one-third of the total patients; those with medial shifts were relatively less. Of the 34 patients, 18 were found to have more than one anomaly preoperatively. Several studies have reported the incidence of a high riding artery to be $\sim 10-31 \%$, which is similar to the results of the present study ${ }^{11,14,15)}$. However, the accuracy of the pedicle screw placement was not found to be related to the $\mathrm{C} 2$ vertebra anomaly.

Out of the total 135 screws, major malposition of grade II and III was found in only three screws. Fortunately, there were no serious complications related to the procedure. Although approximately half of all the patients had C2 vertebrae anom- 

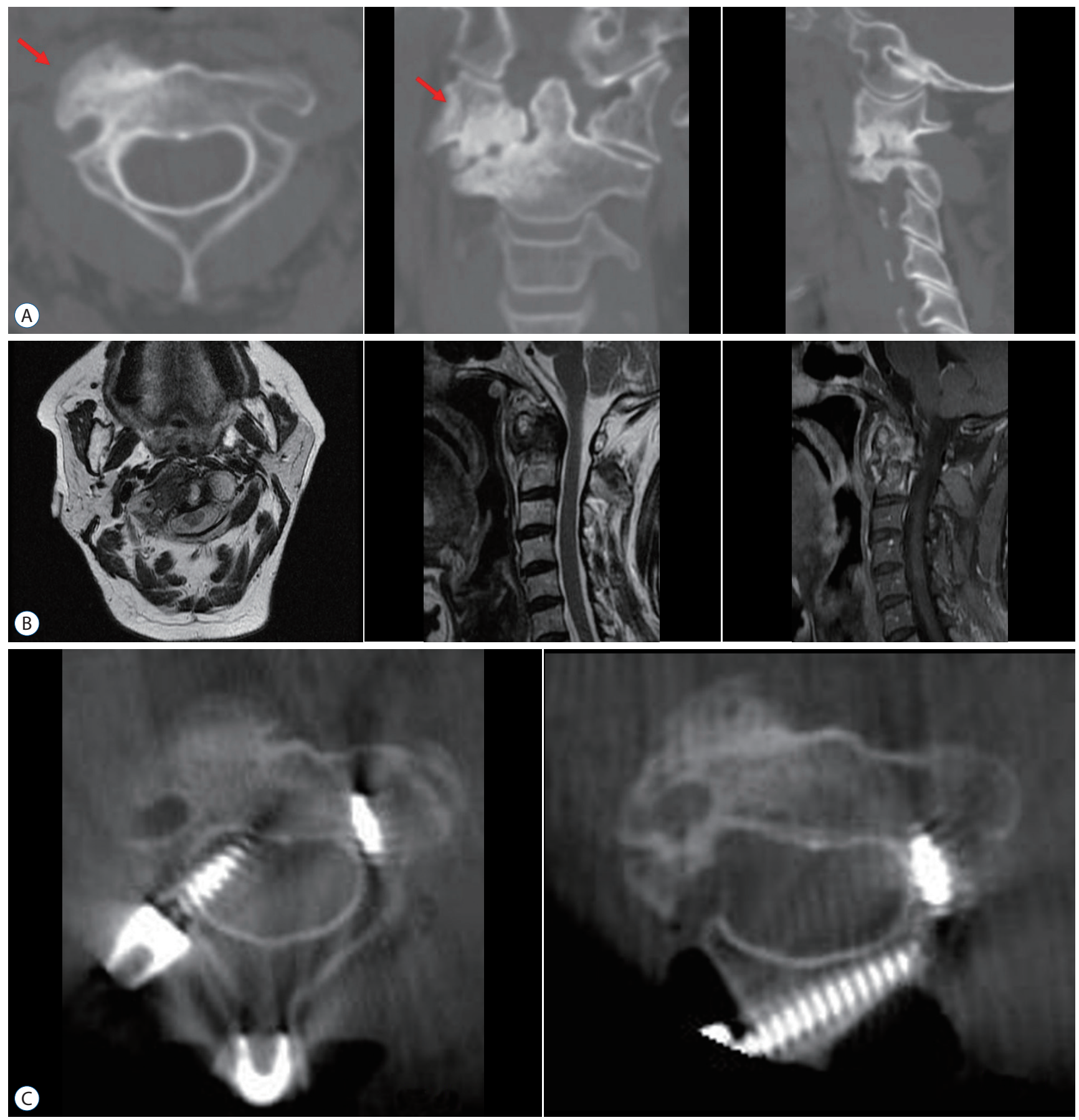

Fig. 3. Cases presentation. A : Preoperative CT images of the $C 2$ vertebra showing $C 1 / 2$ right facet arthropathy with severe sclerotic change (red arrow). B : Preoperative MRI image showing diffuse soft tissue lesion (pannus) around the odontoid process with enhancement. C : With intraoperative CT, we confirmed that the right C2 pedicle screw had invaded into the spinal canal; the right C2 pedicle screw was removed and alternative C2 laminar screw was inserted. CT : computed tomography, MRI : magnetic resonance imaging.

alies, we could avoid the surgical risks through using alternative screws (lamina screw or pars screw). In addition, with intraoperative CT based navigation devices, we could check the trajectory and position of the screw in advance and this seemed to result in better outcomes.

There have been several studies on surgery of the atlantoaxial junction using imaging guided techniques. However, except for case reports, the study by Costa et al. ${ }^{2}$ was the first to 
report on 17 patients with $\mathrm{C} 1-2$ traumatic fractures who underwent surgery using 3D navigation system. Jacob et al. ${ }^{9)}$ also presented similar results with $\mathrm{O}$-arm navigation and analyzed the accuracy of screw placement. Most studies have shown a positive benefit of C1-2 fusion with intraoperative CT based navigation including high accuracy of screw placement and low surgical complications. However, surgery using a navigation device does not always produce good results in atlantoaxial lesions. Hur et al. ${ }^{8)}$ reported on 48 surgically treated patients, which is the largest reported so far, that cortex violation did not completely disappear even after using navigation instruments during pedicle fixation. Hlubek et al. ${ }^{7)}$ compared the accuracy of $\mathrm{C} 2$ pars and pedicle screw placement and concluded that freehand screw placement was more accurate and had a superior result, which was due to an error in the navigation system.

Till date, only a few studies have compared the accuracy of screw placement in atlantoaxial fusion, but much has not been done yet. In our study, we found that operations with $\mathrm{O}$-arm devices slightly improved the accuracy rate of screw positions, but we could not confirm statistically meaningful results.

Surgery for $\mathrm{Cl}-2$ fusion is relatively rare and requires a steep learning curve which is a limitation for large scale studies. Although, the present paper is valuable to assess the usefulness of navigation devices, it did not fully control the variable factors. There is a time gap between the $\mathrm{O}$-arm group and the $\mathrm{C}$ arm group. Since the introduction of the O-arm, only a few Carm-surgeries have been performed except when navigation equipment broke down and could not be used. However, all surgeries were performed with the Goel and Harms technique by experienced spine surgeons and there were no differences between two groups.

There are some concerns while using the intraoperative navigation system. First, it is costly to launch the full navigation system, the scanned image may be distorted due to metal artifacts, and the extra time to set up the devices may prolong the operation time. A blurred image due to metal artifacts can be an obstacle to O-arm surgery. Jacob et al. ${ }^{9)}$ reported difficulty in identifying intraoperative images during dental implants. A similar problem was seen in the present study when confirming images due to barriers such as artificial structures. However, this rarely occurred in the upper cervical spine unlike lower cervical spine surgery. The cervical plate may be the metal artifact which caused most of the problems which the author did not take into account in C1-2 lesions. Some studies that have shown an increased operation time when performing in C1-2 surgery using navigation. Hitti et al. ${ }^{6}$ reported that cervical spine surgery using navigation reduces EBL, but the operation time is prolonged. Unlike the results of previous studies, the present study showed that intraoperative navigation devices can reduced the operative time. However, it could not be concluded that this was due to the navigation device only as the operative time was influenced by many factors such as proficiency of the operator, tendency, and set-up of the operation room. It is expected that more studies will be carried out in the future to verify these conclusions. And comparing the results of operation time of C1-2 fusion with other published papers, we took a longer operative time than other results. We think that the reason was autogenous bone collection of the iliac crest and the meticulous dissection of the surrounding structure to secure the pedicle screw safely. We should take more effort to reduce the operative time.

There are several advantages of using the intraoperative navigation system. First, the accuracy of the pedicle screw placement can be improved by a 3D image of the anatomical structure which enables visualization of any anatomic variations during surgery. This is a feature that is commonly referred to state in studies on intraoperative navigation devices. Second a reduction during the stress of screw fixation, which is the most difficult part of a C1-2 operation, resulted in benefits such as superior surgical outcomes, decrease in the operation time, and decrease in the amount of blood lost. Third, intraoperative navigation devices minimize the radiation exposure to the operator during spinal surgery. This has been reported in several articles, confirming the usefulness of the system ${ }^{1}$. Fourth, the operation results are confirmed in real time, which reduces the possibility of reoperation. As mentioned previously, it is possible to reduce the possibility of reoperation by confirmation of any mal-positioned screws in advance.

In our paper, a total of three surgeons performed the C1-2 fusion surgeries. Of the total 34 cases, senior surgeon performed 31 cases and the other surgeons performed one and two cases, respectively. The other surgeons are relatively beginners with less than 5 years of experience in spinal surgery. In all three cases of the beginner, $\mathrm{O}$-arm instrument was used for surgical treatment. There was a large deviation in the size of each individual surgical case and it was impossible to statis- 
tically derive differences in results from the operators. Because of small cases of beginner operators, it is hard to conclude that the use of $\mathrm{O}$-arm can fully can compensate for the lack of surgical experience.

This study has some limitations. It was a retrospective study and we could not reveal the differences between the two groups in a totally independent manner. There are statistically inconsistent parts of the $\mathrm{O}$-arm and the $\mathrm{C}$-arm groups in some factors. Second, the results of the operation at the initial stage of the surgery with fluoroscopic device and the operation after introducing the intraoperative device might be different. Third, the total number of operators in this paper is three. Because each operator had minimal differences in surgical experience and method, which may lead to errors in the analysis of the surgical results. Fourth, the number of surgical cases is comparatively low, and it is regrettable that the research is not carried out in depth.

\section{CONCLUSION}

In this study, we have reported good surgical results using an intraoperative navigation device in unstable atlantoaxial instability. Comparing to the conventional fluoroscopic guided device, Operation with intraoperative CT guided device had reduced operative time.

\section{CONFLICTS OF INTEREST}

No potential conflict of interest relevant to this article was reported.

\section{INFORMED CONSENT}

This type of study does not require informed consent.

\section{AUTHOR CONTRIBUTIONS}

Conceptualization : JSL, DWS

Data curation : JSL, SHL

Formal analysis : JSL, SW
Funding acquisition : JSL

Methodology : SSK, GSS, DWS

Project administration : JSL

Visualization : SSK, SHL

Writing - original draft : JSL

Writing - review \& editing : JSL, DWS, SHL, SSK, SWL, GSS

\section{ORCID}

Jun Seok Lee https://orcid.org/0000-0003-2488-6953

Dong Wuk Son ～https://orcid.org/0000-0002-9154-1923

Su Hun Lee https://orcid.org/0000-0001-8952-5556

Sung Soon Ki Thtps:/orcid.org/0000-0001-7138-9621

Sang Weon Lee $\quad$ https://orcid.org/0000-0002-3199-7072

Geun Sung Song https://orcid.org/0000-0001-8029-9011

\section{- Acknowledgements}

This study was supported by Research Institute for Convergence of biomedical science and technology Grant (30-2017010), Pusan National University Yangsan Hospital.

\section{References}

1. Abdullah KG, Bishop FS, Lubelski D, Steinmetz MP, Benzel EC, Mroz TE : Radiation exposure to the spine surgeon in lumbar and thoracolumbar fusions with the use of an intraoperative computed tomographic 3-dimensional imaging system. Spine (Phila Pa 1976) 37 : E1074-E1078, 2012

2. Costa F, Ortolina A, Attuati L, Cardia A, Tomei M, Riva M, et al. : Management of C1-2 traumatic fractures using an intraoperative 3D imaging-based navigation system. J Neurosurg Spine 22 : 128-133, 2015

3. Gertzbein SD, Robbins SE : Accuracy of pedicular screw placement in vivo. Spine (Phila Pa 1976) 15 : 11-14, 1990

4. Goel A, Laheri V : Plate and screw fixation for atlanto-axial subluxation. Acta Neurochir (Wien) $129:$ 47-53, 1994

5. Harms J, Melcher RP : Posterior C1-C2 fusion with polyaxial screw and rod fixation. Spine (Phila Pa 1976) 26 : 2467-2471, 2001

6. Hitti FL, Hudgins ED, Chen HI, Malhotra NR, Zager EL, Schuster JM : Intraoperative navigation is associated with reduced blood loss during C1C2 posterior cervical fixation. World Neurosurg 107 : 574-578, 2017

7. Hlubek RJ, Bohl MA, Cole TS, Morgan CD, Xu DS, Chang SW, et al. : Safety and accuracy of freehand versus navigated C2 pars or pedicle screw placement. Spine J 18 : 1374-1381, 2018 
8. Hur JW, Kim JS, Ryu KS, Shin MH : Accuracy and safety in screw placement in the high cervical spine: retrospective analysis of 0 -arm-based navigation-assisted C1 lateral mass and C2 pedicle screws. Clin Spine Surg 32 : 193-199, 2019

9. Jacobs $C$, Roessler PP, Scheidt S, Plöger MM, Jacobs $C$, Disch AC, et al. : When does intraoperative 3D-imaging play a role in transpedicular $\mathrm{C2}$ screw placement? Injury $48:$ 2522-2528, 2017

10. Lee SH, Park DH, Kim SD, Huh DS, Kim KT : Analysis of 3-dimensional course of the intra-axial vertebral artery for $\mathrm{C} 2$ pedicle screw trajectory: a computed tomographic study. Spine (Phila Pa 1976) 39 : E1010E1014, 2014

11. Mandel IM, Kambach BJ, Petersilge CA, Johnstone B, Yoo JU : Morphologic considerations of $\mathrm{C} 2$ isthmus dimensions for the placement of transarticular screws. Spine (Phila Pa 1976) 25 : 1542-1547, 2000

12. Neo M, Sakamoto T, Fujibayashi S, Nakamura T : The clinical risk of vertebral artery injury from cervical pedicle screws inserted in degenerative vertebrae. Spine (Phila Pa 1976) 30 : 2800-2805, 2005

13. Smith JD, Jack MM, Harn NR, Bertsch JR, Arnold PM : Screw placement accuracy and outcomes following 0-arm-navigated atlantoaxial fusion: a feasibility study. Global Spine J 6 : 344-349, 2016

14. Wakao N, Takeuchi M, Nishimura M, Riew KD, Kamiya M, Hirasawa A, et al. : Vertebral artery variations and osseous anomaly at the C1-2 level diagnosed by 3D CT angiography in normal subjects. Neuroradiology $56: 843-849,2014$

15. Yeom JS, Buchowski JM, Kim HJ, Chang BS, Lee CK, Riew KD : Risk of vertebral artery injury: comparison between C1-C2 transarticular and C2 pedicle screws. Spine J $13:$ 775-785, 2013

16. Yukawa $Y$, Kato F, Ito K, Horie Y, Hida T, Nakashima H, et al. : Placement and complications of cervical pedicle screws in 144 cervical trauma patients using pedicle axis view techniques by fluoroscope. Eur Spine J 18 : 1293-1299, 2009 